\title{
Purity of Gaussian states: Measurement schemes and time evolution in noisy channels
}

\author{
Matteo G. A. Paris, ${ }^{1, *}$ Fabrizio Illuminati, ${ }^{2, \dagger}$ Alessio Serafini, ${ }^{2, \$}$ and Silvio De Siena ${ }^{2, \S}$ \\ ${ }^{1}$ Quantum Optics \& Information Group, INFM UdR di Pavia, I-27100 Pavia, Italy \\ ${ }^{2}$ Dipartimento di Fisica "E. R. Caianiello," Università di Salerno, INFM UdR Salerno, INFN Sez. Napoli, Grupo Collegato Salerno, \\ Via S. Allende, 84081 Baronissi (SA), Italy \\ (Received 10 February 2003; revised manuscript received 8 April 2003; published 15 July 2003)
}

\begin{abstract}
We present a systematic study of the purity for Gaussian states of single-mode continuous variable systems. We prove the connection of purity to observable quantities for these states, and show that the joint measurement of two conjugate quadratures is necessary and sufficient to determine the purity at any time. The statistical reliability and the range of applicability of the proposed measurement scheme are tested by means of Monte Carlo simulated experiments. We then consider the dynamics of purity in noisy channels. We derive an evolution equation for the purity of general Gaussian states both in thermal and in squeezed thermal baths. We show that purity is maximized at any given time for an initial coherent state evolving in a thermal bath, or for an initial squeezed state evolving in a squeezed thermal bath whose asymptotic squeezing is orthogonal to that of the input state.
\end{abstract}

DOI: 10.1103/PhysRevA.68.012314 PACS number(s): 03.67.Pp, 03.65.Yz, 42.50.-p, 42.50.Dv

\section{INTRODUCTION}

Nonclassical features of atomic and radiation systems play a relevant role in quantum information, communication, and high precision measurements, as well as in many fundamental experiments to test quantum mechanics $[1,2]$. In particular, pure Gaussian states of continuous variable (CV) systems, such as coherent and squeezed-coherent states, are the key ingredients of secure optical communication [3-6] and Heisenberg limited quantum interferometry [7-11]. The characterization of several properties of Gaussian states has been the subject of intense recent work [12-18], stimulated by the seminal analysis on their entanglement properties $[19,20]$.

Any attempt to exploit Gaussian states for quantum information and quantum measurement schemes must, however, face the obvious difficulty that pure states are unavoidably corrupted by the interaction with the environment. Therefore, CV Gaussian states that are available for experiments are usually mixed states, and it becomes crucial to establish their degree of purity (or mixedness) determined by the environmental noise. In the present paper, we study the purity of Gaussian states for single-mode continuous variable systems focusing on two aspects: its experimental characterization and its time evolution in noisy channels. We first show that the joint detection of two conjugate quadratures is a necessary and sufficient measurement to determine the purity of a Gaussian state with reliable experimental statistics; we then derive an evolution equation for the purity of Gaussian states in a noisy channel, considering the instances of a thermal bath and a squeezed thermal bath, and determine the evolutions that at any given time maximize the purity.

Let us refer to $\mu=\operatorname{Tr}\left[\varrho^{2}\right]$ as to the purity of a generic

\footnotetext{
*Electronic address: paris@unipv.it

†Electronic address: illuminati@sa.infn.it

\#Electronic address: serale@sa.infn.it

${ }^{\S}$ Electronic address: desiena@sa.infn.it
}

quantum state $\varrho$; the conjugate quantity $S_{L}=(1-\mu) d /(d$ -1 ), where $d$ is the dimension of the Hilbert space of the system under investigation, is known as linear entropy or mixedness. In general, $\mu$ ranges from one, which is the value for a pure state, to $\mu=1 / d$, value for a completely mixed state. Throughout the paper, we will consider CV systems, i.e., infinite dimensional Hilbert spaces, and therefore we will have $0<\mu \leqslant 1$. Since $\mu$ is a nonlinear function of the density matrix, it cannot be connected to an observable quantity if we perform repeated measurements on single copies of the state. That is, it cannot be the expectation value of a single-system self-adjoint operator, nor can it be related to a single-system probability distribution obtained from a positive operator-valued measure. On the other hand, if collective measurements on two copies of the state are possible, then the purity may be measured directly [21,22]. For instance, collective measurements of overlap and fidelity have been experimentally realized for qubits encoded into polarization states of photons [23].

In general, purity can be determined by the knowledge of the quantum state of the system, which in turn can be obtained by quantum tomography [24]. However, in this case, the statistics is usually poor, since the measurement of a whole quorum of observables is needed, unavoidably leading to large fluctuations [25]. On the other hand, if we focus our attention on the class of Gaussian states, it is indeed possible to find an operative method to experimentally determine $\mu$. In fact, Gaussian states are uniquely defined by their first two statistical moments, which can be measured by the joint detection of two conjugate quadratures, say position and momentum or quadrature phases of the electromagnetic field. Such a measurement corresponds to an estimate of the $\mathrm{Hu}-$ simi $Q$ function $Q(\alpha)=\langle\alpha|\varrho| \alpha\rangle,|\alpha\rangle$ being a coherent state of the harmonic oscillator. We will show that the measurement of the $Q$ function is the optimal minimal measurement for the purity, in the sense that it is necessary and sufficient to determine $\mu$ and requires the minimum number of observables to be measured.

The joint measurement of two conjugate quadratures is 
possible for a single-mode radiation field as well as for a single atom [26-28]. Remarkably, for these systems, the class of Gaussian states includes almost all the states that can be reliably produced, and employed in communication or measurement protocols.

Finally, we will show that the previous discussion allows us to unravel the dynamics of purity only in terms of observable quantities. Indeed, the time evolution of the purity of an initial Gaussian state in a noisy channel can be uniquely expressed as a function of the initial observable parameters of the input state and of the asymptotic observable parameters of the environment. This property allows us then to determine and engineer optimal evolutions, i.e., evolutions that maximize the purity at any given time.

The paper is structured as follows. In Sec. II, we show how purity is related to observable quantities for Gaussian states, and how it can be obtained either from the $Q$ function or by three single-quadrature detection. In Sec. III, we present the results of a systematic numerical analysis, establishing the statistical reliability and the range of applicability of the method by means of Monte Carlo simulated experimental runs. We also show that the $Q$-function based determination of purity is a more reliable method than the singlequadrature detection. Section IV is devoted to derive and solve an evolution equation for the purity of an initial Gaussian state in a noisy channel, for both thermal and squeezed thermal baths. We show that, even though the asymptotic value of purity is not related to the initial conditions, its behavior at finite times does depend on the initial squeezing and thermal excitations, and we determine the evolutions that maximize the purity at any finite time. We show, in particular, that purity is maximized for an initial coherent state evolving in a thermal bath, or for an initial squeezed state evolving in a squeezed thermal bath whose asymptotic squeezing is orthogonal to that of the input state. Finally, in Sec. V, we present some concluding remarks.

\section{PURITY OF GAUSSIAN STATES}

We begin by reviewing some fundamental properties of the Wigner phase-space representation [29] which will be useful throughout the paper. The Wigner representation of an arbitrary operator $O$ is defined as follows:

$$
O(\alpha)=\int_{\mathrm{C}} \frac{\mathrm{d}^{2} \gamma}{\pi^{2}} e^{\bar{\gamma} \alpha-\gamma \bar{\alpha}} \operatorname{Tr}[O D(\gamma)]
$$

where $D(\gamma)=\exp \left(\gamma a^{\dagger}-\bar{\gamma} a\right)$ is the displacement operator and $\operatorname{Tr}[O D(\gamma)]$ is usually referred to as the characteristic function of the operator $O$. Let $O_{1}$ and $O_{2}$ be operators that admit regular Wigner representations $O_{1}(\alpha)$ and $O_{2}(\alpha)$, respectively. Then, the trace $\operatorname{Tr}\left[O_{1} O_{2}\right]$ can be computed as an integral over phase space according to

$$
\operatorname{Tr}\left[O_{1} O_{2}\right]=\pi \int_{\mathrm{C}} d^{2} \alpha O_{1}(\alpha) O_{2}(\alpha) .
$$

From now on, we will move to the phase-space variables $x$ and $p$, corresponding to quadrature phases $\hat{x}=\left(a+a^{\dagger}\right) / \sqrt{2}$ and $\hat{p}=i\left(a^{\dagger}-a\right) / \sqrt{2}$ of the field $a$, whose expectation values $\langle\hat{x}\rangle \equiv x$ and $\langle\hat{p}\rangle \equiv p$ are related to $\alpha$ by $\alpha=(x+i p) / \sqrt{2}$.

The Wigner representation $W(\alpha)$ of the density matrix $\varrho$ of a quantum state is referred to as the Wigner function of the state. The class of Gaussian states is defined as the class of states with Gaussian Wigner function, namely

$$
W(x, p)=\frac{e^{-1 / 2 X \boldsymbol{\sigma}^{-1} X^{T}}}{\pi \sqrt{\operatorname{Det}[\boldsymbol{\sigma}]}},
$$

where $X$ is the displaced vector $X=\left(x-x_{0}, p-p_{0}\right)$ and $\boldsymbol{\sigma}$ is the covariance matrix

$$
\sigma_{i j}=\frac{1}{2}\left\langle\hat{x}_{i} \hat{x}_{j}+\hat{x}_{j} \hat{x}_{i}\right\rangle-\left\langle\hat{x}_{i}\right\rangle\left\langle\hat{x}_{j}\right\rangle
$$

where $\hat{x}_{1}=\hat{x}$ and $\hat{x}_{2}=\hat{p}$. The density matrix of the most general Gaussian state can be written as [30]

$$
\varrho=D\left(\alpha_{0}\right) S(r, \varphi) \nu_{n}^{-} S^{\dagger}(r, \varphi) D^{\dagger}\left(\alpha_{0}\right),
$$

where $\alpha_{0}=\left(x_{0}+i p_{0}\right) / \sqrt{2}, \nu_{n}^{-}$is a thermal state with average photon number $\bar{n}$,

$$
\nu_{n}^{-}=\frac{1}{1+\bar{n}} \sum_{k=0}^{\infty}\left(\frac{\bar{n}}{1+\bar{n}}\right)^{k}|k\rangle\langle k|,
$$

$D\left(\alpha_{0}\right)$ denotes the displacement operator, and $S(r, \varphi)$ $=\exp \left(\frac{1}{2} r e^{-i 2 \varphi} a^{2}-\frac{1}{2} r, e^{i 2 \varphi} a^{\dagger 2}\right)$ is the squeezing operator. A convenient parametrization of Gaussian states can be achieved by replacing the $\sigma_{i j}$ 's with $n, r, \varphi$, which have a more direct phenomenological interpretation. By applying the phasespace representation of squeezing [29,31], the following relations are easily derived:

$$
\begin{aligned}
\sigma_{x x} & =\frac{2 \bar{n}+1}{2}[\cosh (2 r)-\sinh (2 r) \cos (2 \varphi)], \\
\sigma_{p p} & =\frac{2 \bar{n}+1}{2}[\cosh (2 r)+\sinh (2 r) \cos (2 \varphi)], \\
\sigma_{x p} & =\frac{2 \bar{n}+1}{2} \sinh (2 r) \sin (2 \varphi) .
\end{aligned}
$$

Exploiting Eq. (2), one can write

$$
\mu \doteq \operatorname{Tr}\left[\varrho^{2}\right]=\frac{\pi}{2} \int_{\mathbb{R}} \int_{\mathbb{R}} d x d p W^{2}(x, p),
$$

so that for a Gaussian state,

$$
\mu=\frac{1}{2 \sqrt{\operatorname{Det}[\boldsymbol{\sigma}]}}=\frac{1}{2 \sqrt{\sigma_{x x} \sigma_{p p}-\sigma_{x p}^{2}}} .
$$

In terms of $\bar{n}, r$, and $\varphi$, Eq. (7) can then be recast as $[32,33]$ 


$$
\mu=\frac{1}{2 \bar{n}+1} .
$$

Equation (8) shows that the purity of a generic Gaussian state depends only on the average number of thermal photons, as one should expect, since displacement and squeezing are unitary operations. Therefore, the measurement of the purity of a Gaussian state is equivalent to the measurement of its average number of thermal photons.

As the last step in connecting $\mu$ to observables, we report the expression of the $\sigma_{i j}$ 's in terms of the $Q$ function $Q(\alpha)$. This follows from the antinormally ordered expression of the second moments. We have, for instance,

$$
\hat{x}^{2}=\frac{a^{2}+a^{\dagger 2}+2 a a^{\dagger}-\mathbb{I}}{2},
$$

which, in terms of phase-space variables, corresponds to $x^{2}$ $-\frac{1}{2}$. Therefore, we eventually get

$$
\left\langle\hat{x}^{2}\right\rangle=\operatorname{Tr}\left[\varrho \frac{\left(a+a^{\dagger}\right)^{2}}{2}\right]=\int_{\mathbb{R}} \int_{\mathbb{R}} d x d p Q(x, p)\left(x^{2}-\frac{1}{2}\right),
$$

where we have moved from variables $\alpha$ and $\bar{\alpha}$ to variables $x$ and $p$, previously defined. In much the same way, we obtain

$$
\begin{aligned}
& \left\langle\hat{p}^{2}\right\rangle=\int_{\mathbb{R}} \int_{\mathbb{R}} d x d p Q(x, p)\left(p^{2}-\frac{1}{2}\right), \\
& \frac{1}{2}\langle\hat{x} \hat{p}+\hat{p} \hat{x}\rangle=\int_{\mathbb{R}} \int_{\mathbb{R}} d x d p Q(x, p) x p .
\end{aligned}
$$

Since first moments are naturally antinormally ordered, evaluation of first moments of quadratures is easily obtained, and the $\sigma_{i j}$ 's can be eventually computed.

Gaussian states may be effectively characterized as well by single-quadrature measurements obtained by balanced homodyne detection [34]. Thus, a question arises whether or not one really needs to resort to joint measurement of two conjugate quadratures to determine the purity. In particular, since Gaussian states are fully characterized by the first and second moments, it suffices to measure the rotated quadrature $x_{\theta}=\left(a^{\dagger} e^{i \theta}+a e^{-i \theta}\right) / \sqrt{2}$ for three different values of $\theta$ to have a complete characterization of the state, including the measure of its purity. This fact can be proven by reminding that the probability distribution $p(x, \theta)$ of a measurement of $x_{\theta}$ on a state of the form (4) is a Gaussian centered in $x_{0}$ $=\operatorname{Re}\left[\alpha_{0} e^{-i \theta}\right]$, with variance

$$
\sigma_{\theta}=\frac{1}{2 \mu}\left[e^{-2 r} \cos ^{2}(\theta-\varphi)+e^{2 r} \sin ^{2}(\theta-\varphi)\right] .
$$

By measuring three quadratures, we directly obtain the purity $\mu$ by comparison of variances. By choosing $\theta=0, \pi / 2, \pi / 4$, we have

$$
\mu=\left[4 \sigma_{\pi / 4}\left(\sigma_{0}+\sigma_{\pi / 2}-\sigma_{\pi / 4}\right)-\left(\sigma_{0}-\sigma_{\pi / 2}\right)^{2}\right]^{-1 / 2} .
$$

In the following section, we will compare the two different experimental schemes on the basis of Monte Carlo simulated experiments.

\section{MONTE CARLO SIMULATED EXPERIMENTS}

As we have seen, in order to evaluate the $\sigma_{i j}$ 's and then the purity, we need to estimate averages over the $Q$ function. These estimates can be obtained if one disposes of data distributed according to the $Q$ function $Q(x, p)$ itself. Indeed, such a distribution can be experimentally reconstructed for a single-mode radiation field through heterodyne [35], eightport homodyne [36,37], or six-port homodyne detectors [38], and for atoms by coupling the atom with two light fields and measuring the corresponding phase shifts [28].

In order to test the effectiveness of the proposed scheme, we have performed a systematic numerical analysis by means of Monte Carlo simulated experiments. The simulations are needed to show the actual independence of the method on the squeezing and displacing parameters, in compliance with Eq. (8). Moreover, they provide a crucial test on the actual possibility of getting reliable (i.e., with reduced fluctuations) determinations of $\mu$ in realistic experimental settings and even for most unfavorable states.

The purity $\mu$ and its dispersion $\Delta \mu$ have been evaluated from samples of the $Q$ function, varying the values of the parameters of the simulated Gaussian state. Besides $\bar{n}, r, \varphi$, and $\alpha_{0}$, the experimental determination of the $Q$ function depends on the number $N_{x}$ of collected data.

We find that $\mu$ and $\Delta \mu$ are essentially independent of the complex displacement parameter $\alpha_{0}$ and squeezing angle $\varphi$. On the other hand, $\Delta \mu$ does depend on $\bar{n}$ and $r$, decreasing with increasing $\bar{n}$ and increasing with increasing $r$.

In Fig. 1, we report the determination of purity for a strongly squeezed thermal state as a function of the number of data. The error on purity is of the order of a few percent for samples made of $N_{x} \simeq 10^{5}$ data.

In order to compare the determination of $\mu$ by the $Q$ function with that coming from single-quadrature detection, we have simulated the measurement of three quadratures $x_{\theta}$, $\theta=0, \pi / 2, \pi / 4$ by balanced homodyne detection. In Fig. 2 , we report the estimated purity [using Eq. (12)] for the same strongly squeezed thermal state of Fig. 1 as a function of the number of data. Some features are immediately evident. First of all, one can see that the determination is biased: in the present case, the estimated $\mu$ is always larger than the true value, while the opposite case occurs by inverting the phase of the squeezing. Therefore, the method is very sensitive to the choice of the phase. Moreover, the relative error is not a smooth function of the number of data, i.e., the method is not statistically reliable as the joint-measurement one. This is again due to the remarkable dependence of the variances on the phase of the squeezing, a dependence which is instead smoothed out in the measurement of the $Q$ function. Summing up, for some specific states (as the example considered here) single-quadrature detection may be asymptotically even more efficient than the heterodyne one. However, in general, the number of data needed for the relative error to be 

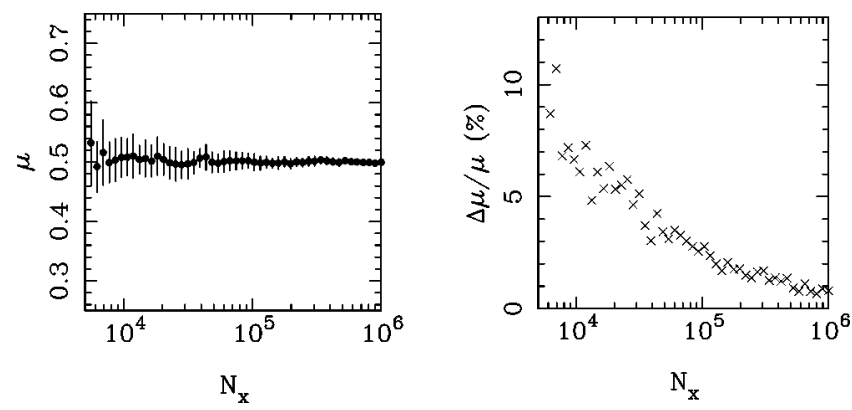

FIG. 1. Effect of the number of data on the $Q$-function based determination of purity for Gaussian states: results from Monte Carlo simulated experiments. In the left graph, we plot the determination of the purity $\mu$ versus the number of data $N_{x}$ for a squeezed thermal state with parameters given by $\alpha_{0}=0, \varphi=0$, and $r=1.5$, corresponding to $\sinh ^{2} 1.5 \simeq 4.5$ mean squeezed photons, and a mean number of thermal photons $\bar{n}=0.5$. Black circles are the estimated values of purity based on the $Q$-distributed statistics, vertical bars are the experimental errors (confidence intervals); for a large number of experimental data, the errors quickly fall well within the black circles of the estimated values. The theoretical value of purity for all the simulated experimental runs is $\mu=0.5$. In the right graph, we report the relative errors $\Delta \mu / \mu$ versus the number of data for the same squeezed thermal state.

below the joint-measurement level is strongly state dependent. We conclude that the measurement of the $Q$ function is statistically more reliable and thus more suited for a systematic analysis of the purity of Gaussian states.

Let us now go back to the analysis of the $Q$-function determination of purity. A smaller number of data is needed to obtain a given precision for states with smaller squeezing. The effect of the squeezing parameter on the determination
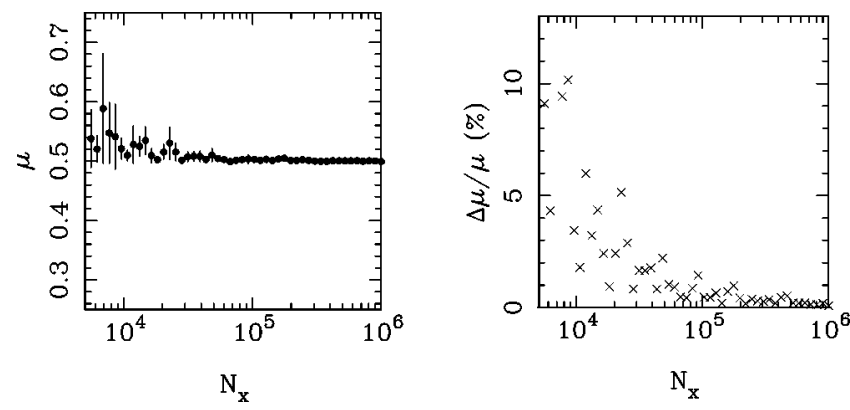

FIG. 2. Effect of the number of data on the determination of purity for Gaussian states by single-quadrature detection: results from Monte Carlo simulated experiments of three quadratures $x_{0}$, $x_{\pi / 2}$, and $x_{\pi / 4}$. In the left graph, we plot the determination of the purity $\mu$ versus the number of data $N_{x}$ for the same squeezed thermal state of Fig. 1. Note that, in this instance, the total number of data $N_{x}$ corresponds to $N_{x} / 3$ detections for each quadrature. Black circles are the estimated values of purity based on the balanced homodyne statistics, vertical bars are the experimental errors (confidence intervals); for a large number of experimental data the errors quickly fall well within the black circles of the estimated values. The theoretical value of purity for all the simulated experimental runs is $\mu=0.5$. In the right graph, we report the relative errors $\Delta \mu / \mu$ versus the number of data for the same squeezed thermal state.
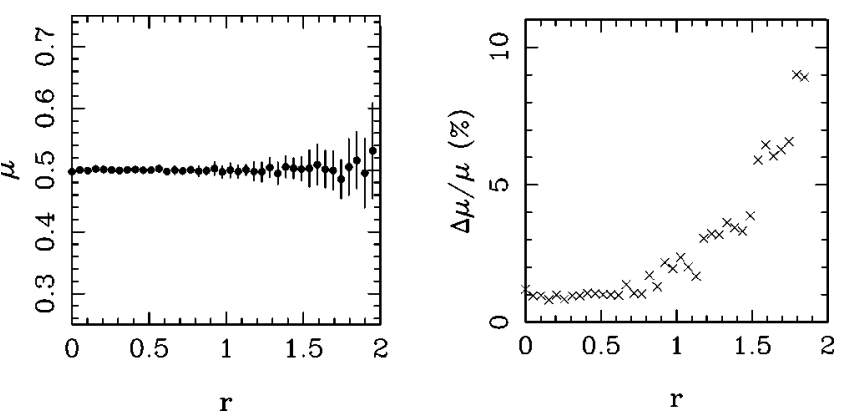

FIG. 3. Effect of squeezing on the $Q$-function based determination of purity for Gaussian states: results from Monte Carlo simulated experiments. In the left graph, we plot the determination of purity versus the squeezing parameter $r$ for Gaussian states with the other parameters fixed at $\alpha=0, \varphi=0$, and $\bar{n}=0.5$. Black circles are the determined values of purity based on the $Q$-distributed statistics, vertical bars are the experimental errors (confidence intervals). For small $r$, the errors are within the black circles. The theoretical value of purity for all the states is $\mu=0.5$. In the right graph, we report the relative errors $\Delta \mu / \mu$ versus the squeezing parameter for the same set of experiments. The number of data in all simulated experiments is $N_{x}=3 \times 10^{4}$.

of purity is illustrated in Fig. 3, where we report $\mu$ and $\Delta \mu / \mu$ versus $r$ for Gaussian states with $\alpha=0, \varphi=0$, and $\bar{n}=0.5$, and for a number of data $N_{x}=3 \times 10^{4}$. Notice that, in Fig. 3, the range of $r$ corresponds to quite a large number of mean squeezed photons $0 \leqslant \sinh ^{2} r \lesssim 15$.

In the deep quantum regime, i.e., for small $\bar{n}$, fluctuations of $\mu$ become more relevant. This is not surprising, since $\mu$ is a highly nonlinear function of the second-order moments. However, simulations show that even for highly squeezed (up to $\simeq 15$ mean squeezed photons) and slightly mixed (down to $\bar{n} \simeq 0.1$ ) states, realistic experimental conditions allow a statistically reliable determination of $\mu$ that complies with the theoretical expectation (8), up to an error of a few percent. In Fig. 4, we plot the determination of purity for different squeezed thermal states as a function of the average number of thermal photons $\bar{n}$, for samples made of $N_{x}$ $=10^{5}$ data.

From the above analysis, we conclude that the joint measurement of two conjugate quadratures provides a statistically reliable method to determine the purity of a generic Gaussian state. This is best achieved with experimental schemes that involve data distributed according to the $\mathrm{Hu}-$ simi $Q$ function, such as heterodyne and multiport homodyne detection schemes.

\section{EVOLUTION OF PURITY IN A NOISY CHANNEL}

Let us consider the time evolution of an initial, pure or mixed, generic single-mode Gaussian state in the presence of noise and damping (and/or pumping) toward a final squeezed thermal state. If $\Gamma^{-1}$ is the photon lifetime in the noisy channel, the evolution of a state is described, in the interaction picture, by the following master equation: 

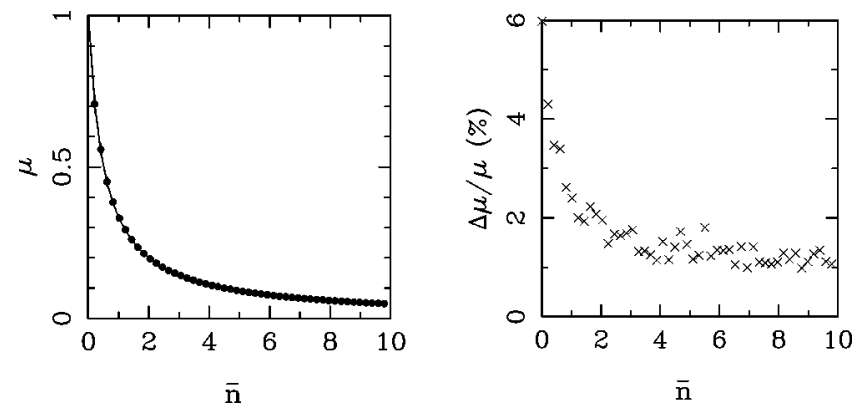

FIG. 4. Effect of thermal photons on the $Q$-function based determination of purity for Gaussian states: results from Monte Carlo simulated experiments. In the left graph, we plot the determination of purity versus the value of $\bar{n}$ for Gaussian states with the other parameters fixed at $\alpha=0, \varphi=0$, and $r=1.0$. Black circles are the determined values of purity according to the $Q$-function statistics, and vertical bars denote the experimental errors (confidence intervals); the latter are within the black circles essentially for all values of $\bar{n}$. The solid line reports the theoretical values of $\mu$. In the right graph, we report the relative errors $\Delta \mu / \mu$ versus $\bar{n}$ for the same set of experiments. The number of data in all simulated experiments is $N_{x}=10^{4}$.

$$
\begin{aligned}
\dot{\varrho}= & \frac{\Gamma}{2} N L\left[a^{\dagger}\right] \varrho+\frac{\Gamma}{2}(N+1) L[a] \varrho \\
& -\frac{\Gamma}{2}\left(\bar{M} D[a] \varrho+M D\left[a^{\dagger}\right] \varrho\right),
\end{aligned}
$$

where the dot stands for time derivative and the Lindblad superoperators are defined by

$$
\begin{gathered}
L[O] \varrho \equiv 2 O \varrho O^{\dagger}-O^{\dagger} O \varrho-\varrho O^{\dagger} O \\
D[O] \varrho \equiv 2 O \varrho O-O O \varrho-\varrho O O .
\end{gathered}
$$

$M$ is the correlation function of the bath (which is usually referred to as the squeezing of the bath); it is, in general, a complex number $M=M_{1}+i M_{2}$, and $\bar{M}$ denotes its complex conjugate, while $N$ is a phenomenological parameter related, as we shall see, to the purity of the asymptotic state. Positivity of the density matrix imposes the important constraint $|M|^{2} \leqslant N(N+1)$. At thermal equilibrium, i.e., for $M=0, N$ coincides with the average number of thermal photons in the bath. The master equation (13) can be transformed into a Fokker-Planck equation for the Wigner function $W(x, p, t)$. Using the differential representation of the superoperators $[31,39]$ in Eq. (13), the corresponding Fokker-Planck equation reads as follows:

$$
\begin{aligned}
\dot{W}(x, p, t)= & \frac{\Gamma}{2}\left(2+x \partial_{x}+p \partial_{p}+\frac{2 N+1}{2}\left(\partial_{x x}^{2}+\partial_{p p}^{2}\right)\right. \\
& \left.+M_{1}\left(\partial_{x x}^{2}-\partial_{p p}^{2}\right)+2 M_{2} \partial_{x p}\right) W(x, p, t) .
\end{aligned}
$$

For a general single-mode Gaussian state of the form (3), one has, in compact notation,

$$
\begin{aligned}
\dot{W}= & \frac{\Gamma}{2}\left(2-X \boldsymbol{\sigma}^{-1}\left(\begin{array}{l}
x \\
p
\end{array}\right)+\frac{2 N+1}{2} \mathcal{S}_{X \boldsymbol{\sigma}}(\mathrm{I})\right. \\
& \left.+M_{1} \mathcal{S}_{X \boldsymbol{\sigma}}(\mathrm{A})+M_{2} \mathcal{S}_{X \boldsymbol{\sigma}}(\mathrm{B})\right) W
\end{aligned}
$$

where $\mathcal{S}_{X \boldsymbol{\sigma}}(\boldsymbol{\gamma})$ denotes the seralian (or siralian) operator, a scalar function of the matrix $\gamma$ given by $\mathcal{S}_{X \boldsymbol{\sigma}}(\boldsymbol{\gamma})$ $\equiv X \boldsymbol{\sigma}^{-1} \boldsymbol{\gamma} \boldsymbol{\sigma}^{-1} X^{T}-\operatorname{Tr}\left[\boldsymbol{\gamma} \boldsymbol{\sigma}^{-1}\right]$. The displaced vector $X$ and the covariance matrix $\boldsymbol{\sigma}$ have been previously defined, whereas $\mathbb{I}, A, B$ form a basis in the space of $2 \times 2$ real symmetric matrices:

$$
\begin{gathered}
I=\left(\begin{array}{ll}
1 & 0 \\
0 & 1
\end{array}\right), \\
A=\left(\begin{array}{cc}
1 & 0 \\
0 & -1
\end{array}\right), \\
B=\left(\begin{array}{ll}
0 & 1 \\
1 & 0
\end{array}\right) .
\end{gathered}
$$

For any given real matrix $\boldsymbol{\gamma}$ and generic Gaussian states, the seralian operator shows the remarkable property

$$
\int_{\mathbb{R}} d x \int_{\mathbb{R}} d p \mathcal{S}_{X \boldsymbol{\sigma}}(\boldsymbol{\gamma}) W(x, p, t)=0
$$

It can be easily shown that this property assures that the last three terms of Eq. (17) [corresponding to diffusion terms in the Fokker-Planck equation (16)] do not enter in the timeevolution equations for the first statistical moments $x_{0}$ and $p_{0}$. Such an evolution is governed by the drift terms and is described by the following equation for the vector $X_{0} \equiv\left(\begin{array}{c}x_{0} \\ p_{0}\end{array}\right)$

$$
\dot{X}_{0}=\int_{\mathbb{R}} d x \int_{\mathbb{R}} d p\left(\begin{array}{l}
x \\
p
\end{array}\right) \frac{\dot{W}}{2}=-\frac{\Gamma}{2} X_{0} .
$$

First moments are damped through the noisy channel: this effect should be expected since it is the mathematical evidence of the absorption of the state's coherent photons.

The evolution of the covariance matrix of the state can be described by monitoring different sets of variables. A good choice of variables is given by the $\sigma_{i j}$ 's, in terms of which the evolution equations decouple. The relations between the variables $\sigma_{i j}$ and the variables $\mu, r$, and $\varphi$ are given in Eqs. (5) and (7). Here we recall some further relations that will be useful in the following:

$$
\operatorname{Det}[\boldsymbol{\sigma}]=\sigma_{x x} \sigma_{p p}-\sigma_{x p}^{2}=\frac{(2 \bar{n}+1)^{2}}{4}=\frac{1}{4 \mu^{2}},
$$




$$
\begin{gathered}
\operatorname{Tr}[\boldsymbol{\sigma}]=\sigma_{x x}+\sigma_{p p}=(2 \bar{n}+1) \cosh (2 r)=\frac{\cosh (2 r)}{\mu} \\
\sigma_{p p}-\sigma_{x x}=\frac{\sinh (2 r) \cos (2 \varphi)}{\mu}
\end{gathered}
$$

As we have seen, in the Wigner phase-space picture, the expectation values can be computed as phase-space integrals. The first-order evolution equation for the covariance matrix $\boldsymbol{\sigma}$ is thus obtained by a straightforward integration, and reads

$$
\dot{\boldsymbol{\sigma}}=\Gamma\left(\boldsymbol{\sigma}_{\infty}-\boldsymbol{\sigma}\right)
$$

with

$$
\boldsymbol{\sigma}_{\infty} \equiv\left(\begin{array}{cc}
\frac{(2 N+1)+2 M_{1}}{2} & M_{2} \\
M_{2} & \frac{(2 N+1)-2 M_{1}}{2}
\end{array}\right)
$$

The matrix $\boldsymbol{\sigma}_{\infty}$, determined by the bath parameters alone, turns out to be the asymptotic covariance matrix. In fact, integration of Eq. (23) yields

$$
\boldsymbol{\sigma}(t)=\boldsymbol{\sigma}_{\infty}\left(1-e^{-\Gamma t}\right)+\boldsymbol{\sigma}(0) e^{-\Gamma t} .
$$

Equation (25) shows a simple example of a Gaussian, completely positive map [40]. The Gaussian character of the evoluted Wigner function can be proven, a posteriori, by verifying that a function of the form (3), with the covariance matrix given by Eq. (25), indeed solves Eq. (16). In order to be a bona fide covariance matrix, $\boldsymbol{\sigma}(t)$ must satisfy the usual condition encoding the $\hat{x}-\hat{p}$ uncertainty relations $[40,41]$ :

$$
\boldsymbol{\sigma}(t)+\frac{i}{2} \mathbf{J} \geqslant 0
$$

with

$$
\mathbf{J}=\left(\begin{array}{cc}
0 & 1 \\
-1 & 0
\end{array}\right)
$$

It is promptly seen that such a condition is satisfied at any time by the convex combination giving $\boldsymbol{\sigma}(t)$ in Eq. (25), if and only if $\boldsymbol{\sigma}_{\infty}$ is a legitimate covariance matrix. This last requirement is assured by the necessary constraint $N(N$ $+1) \geqslant|M|^{2}$ that guarantees positivity of the density matrix.

By introducing

$$
\mu_{\infty} \doteq\left[(2 N+1)^{2}-4|M|^{2}\right]^{-1 / 2}
$$

and exploiting Eqs. (20)-(22), we can eventually express $\mu$, $r$ and $\varphi$ as functions of time:

$$
\begin{aligned}
\mu(t)= & \mu_{0}\left[\frac{\mu_{0}^{2}}{\mu_{\infty}^{2}}\left(1-e^{-\Gamma t}\right)^{2}+e^{-2 \Gamma t}\right. \\
& +2 \mu_{0}\left(\frac{\sqrt{1+4 \mu_{\infty}^{2}|M|^{2}} \cosh \left(2 r_{0}\right)}{\mu_{\infty}}+2 \sinh \left(2 r_{0}\right)\right. \\
& \left.\times\left[M_{1} \cos \left(2 \varphi_{0}\right)-M_{2} \sin \left(2 \varphi_{0}\right)\right]\right) \\
& \left.\times\left(1-e^{-\Gamma t}\right) e^{-\Gamma t}\right]^{-1 / 2}, \\
\cosh [2 r(t)]=\mu(t)\left(\frac{\sqrt{1+4 \mu_{\infty}^{2}|M|^{2}}\left(1-e^{-\Gamma t}\right)}{\mu_{\infty}}\right. & \left.+e^{-\Gamma t} \frac{\cosh \left(2 r_{0}\right)}{\mu_{0}}\right)
\end{aligned}
$$

$\tan [2 \varphi(t)]$

$$
=\frac{M_{2} 2 \mu_{0}\left(1-e^{-\Gamma t}\right)+\sinh \left(2 r_{0}\right) \sin \left(2 \varphi_{0}\right) e^{-\Gamma t}}{-M_{1} 2 \mu_{0}\left(1-e^{-\Gamma t}\right)+\sinh \left(2 r_{0}\right) \cos \left(2 \varphi_{0}\right) e^{-\Gamma t}},
$$

where $\mu_{0}, r_{0}$, and $\varphi_{0}$ are, respectively, the initial purity and the initial squeezing parameters.

Let us first consider the case $M=0$, for which the initial state is damped toward a thermal state with mean photon number $N[29,42]$. In this case, see Eq. (29), $\varphi$ is constant in time and does not enter in the expression of $\mu$. The corresponding solutions for $\mu(t)$ and $r(t)$ then read as follows:

$$
\begin{aligned}
& \mu(t)= \mu_{0}\left[\frac{\mu_{0}^{2}}{\mu_{\infty}^{2}}\left(1-e^{-\Gamma t}\right)^{2}+2 \frac{\mu_{0}}{\mu_{\infty}} e^{-\Gamma t}\left(1-e^{-\Gamma t}\right)\right. \\
&\left.\times \cosh \left(2 r_{0}\right)+e^{-2 \Gamma t}\right]^{-1 / 2}, \\
& \cosh [2 r(t)]=\mu(t)\left(\frac{1-e^{-\Gamma t}}{\mu_{\infty}}+e^{-\Gamma t} \frac{\cosh \left(2 r_{0}\right)}{\mu_{0}}\right) .
\end{aligned}
$$

The quantities $\mu(t)$ and $r(t)$ in Eqs. (30) and (31) solve the following system of coupled equations:

$$
\begin{gathered}
\dot{\mu}=\Gamma\left(\mu-\frac{\mu^{2} \cosh (2 r)}{\mu_{\infty}}\right), \\
\dot{r}=-\frac{\Gamma}{2} \frac{\mu}{\mu_{\infty}} \sinh (2 r),
\end{gathered}
$$

which, in turn, can be directly found by working out the basic evolution equation $\dot{\mu}=2 \operatorname{Tr}[\dot{\varrho} \varrho]$ as a phase-space integral and exploiting Eqs. (20)-(22). It is easy to see that, as $t \rightarrow \infty, \mu(t) \rightarrow \mu_{\infty}=(2 N+1)^{-1}$ and $r(t) \rightarrow 0$, as one expects, since the channel damps (pumps) the initial state to a thermal 


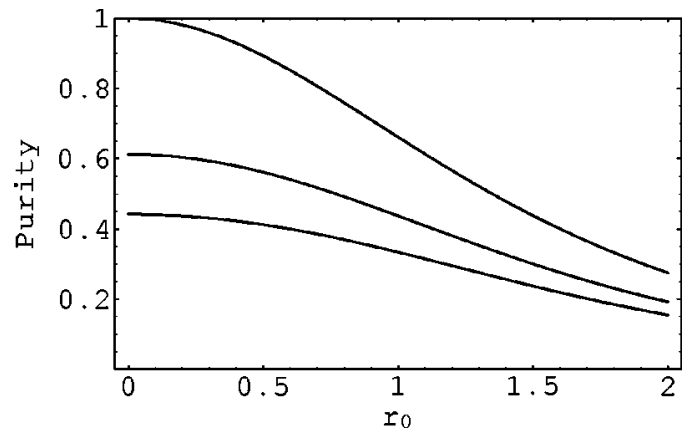

FIG. 5. Plot of the purity $\mu$ for an initially pure Gaussian state $\left(\mu_{0}=1\right)$ in different nonsqueezed $(M=0)$ noisy channels, evaluated at time $t=\Gamma^{-1}$, as a function of the initial squeezing parameter $r_{0}$. From top to bottom, the value of the mean thermal photon number $N$ that characterizes the different channels is $N=0, N$ $=0.5$, and $N=1$, respectively.

state with mean photon number $N$. Therefore, the only constant solution of Eq. (32) is $\mu=\mu_{\infty}, r=0$, i.e., only initial nonsqueezed states are left unchanged by the evolution in the noisy channel. In fact, Eq. (30) shows that $\mu(t)$ is a decreasing function of $r_{0}$ : in a nonsqueezed channel $(M=0)$, a squeezed state decoheres more rapidly than a non-squeezed one (see Figs. 5 and 6). Let us consider, for instance, an initially pure state in a channel with $N=1$ (so that $\mu_{\infty}$ $=\frac{1}{3}$ ); after a time $t=\Gamma^{-1}$, the ratio of the purity of a state with $r_{0}=1.5$ to the purity of a state with $r_{0}=0$ is $53.7 \%$. This dependence could therefore be relevant for practical purposes. The optimal evolution for the purity, obtained letting $r=0$ in Eq. (30), reads

$$
\mu(t)=\frac{\mu_{0} \mu_{\infty}}{\mu_{0}+e^{-\Gamma t}\left(\mu_{\infty}-\mu_{0}\right)} .
$$

Obviously, $\mu(t)$ is not necessarily a decreasing function of time. If $\mu_{0}<\mu_{\infty}$, then the initial state will undergo a certain amount of purification, asymptotically reaching the value $\mu_{\infty}$ which characterizes the channel, as shown in Fig.

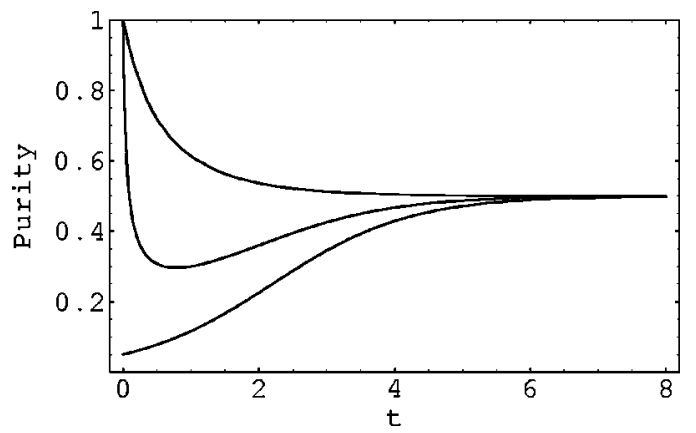

FIG. 6. The purity $\mu$ for various Gaussian states evolving in a channel with $N=0.5, M=0$, as a function of time. Time is dimensionless and measured in units of $\Gamma^{-1}$. The upper curve refers to an initial pure coherent state $\left(r_{0}=0, \mu_{0}=1\right)$, the central curve to an initial pure squeezed vacuum $\left(r_{0}=1.5, \mu_{0}=1\right)$, and the lower curve to an initial thermal state with $r_{0}=0$ and $\mu_{0}=0.05$, i.e., $\bar{n}_{0}$ $=9.5$.
6. In addition, $\mu(t)$ is not a monotonic function for any choice of the initial conditions. Letting $\dot{\mu}=0$ in Eq. (32), and exploiting Eqs. (30) and (31), one finds the following condition for the appearance of a zero of $\dot{\mu}$ at finite positive times: $\cosh \left(2 r_{0}\right)>\max \left(\mu_{0} / \mu_{\infty}, \mu_{\infty} / \mu_{0}\right)$. If this condition is satisfied, then $\mu(t)$ shows a local extremum, in fact a minimum, since differentiating the first of Eqs. (32) and letting $\dot{\mu}=0$, one obtains $\ddot{\mu}>0$. This behavior is shown in Fig. 6 .

Let us now treat the more general instance $M \neq 0$ of a squeezed thermal bath. Recalling the definition of $\mu_{\infty}$ and exploiting Eqs. (27)-(29), one easily finds the asymptotic values of the physical parameters $\mu, r$, and $\varphi$ :

$$
\begin{gathered}
\mu_{\infty}=\frac{1}{\sqrt{(2 N+1)^{2}-4|M|^{2}}}, \\
\cosh \left(2 r_{\infty}\right)=\sqrt{1+4 \mu_{\infty}^{2}|M|^{2}}, \\
\tan \left(2 \varphi_{\infty}\right)=-\frac{M_{2}}{M_{1}} .
\end{gathered}
$$

These values characterize the squeezed channel. Equation (34) shows that if $M \neq 0$, then $N$ is not simply the mean thermal photon number $\bar{n}$ of the asymptotic state. One has

$$
N=\frac{\sqrt{(2 \bar{n}+1)^{2}+4|M|^{2}}-1}{2} .
$$

In order to understand the dynamics of purity when $M$ $\neq 0$, it is convenient to write again the expression (27) for $\mu(t)$, using Eqs. (35) and (36) to switch from the complex parameter $M=M_{1}+i M_{2}$ to the asymptotic values of the squeezing parameters $r_{\infty}$ and $\varphi_{\infty}$; one obtains

$$
\begin{aligned}
\mu(t)= & \mu_{0}\left[\frac{\mu_{0}^{2}}{\mu_{\infty}^{2}}\left(1-e^{-\Gamma t}\right)^{2}+e^{-2 \Gamma t}\right. \\
& +2 \frac{\mu_{0}}{\mu_{\infty}}\left\{\cosh \left(2 r_{\infty}\right) \cosh \left(2 r_{0}\right)+\sinh \left(2 r_{\infty}\right) \sinh \left(2 r_{0}\right)\right. \\
& \left.\left.\times\left[\cos \left(2 \varphi_{\infty}-2 \varphi_{0}\right)\right]\right\}\left(1-e^{-\Gamma t}\right) e^{-\Gamma t}\right]^{-1 / 2}
\end{aligned}
$$

We see from Eq. (37) that $\mu(t)$ is a monotonically decreasing function of the factor $\cos \left(2 \varphi_{\infty}-2 \varphi_{0}\right)$, which gives the only dependence on the initial phase $\varphi_{0}$ of the squeezing. Thus, for any given $\varphi_{\infty}$ characterizing the squeezing of the bath, $\varphi_{0}=\varphi_{\infty}+\pi / 2$ is the most favorable value of the initial angle of squeezing, i.e., the one which allows the maximum purity at a given time. For such a choice, $\mu(t)$ reduces to

$$
\begin{aligned}
\mu(t)= & \mu_{0}\left[\frac{\mu_{0}^{2}}{\mu_{\infty}^{2}}\left(1-e^{-\Gamma t}\right)^{2}+e^{-2 \Gamma t}+2 \frac{\mu_{0}}{\mu_{\infty}}\right. \\
& \left.\times \cosh \left(2 r_{\infty}-2 r_{0}\right)\left(1-e^{-\Gamma t}\right) e^{-\Gamma t}\right]^{-1 / 2} .
\end{aligned}
$$


This is a decreasing function of the factor $\cosh \left(2 r_{\infty}-2 r_{0}\right)$, so that the maximum value of the purity at a given time is achieved for the choice $r_{0}=r_{\infty}$, and the evolution of the purity of a squeezed state in a squeezed channel is identical to the evolution of the purity of a nonsqueezed state in a nonsqueezed channel expressed by Eq. (33) and illustrated in Fig. 6.

In conclusion, for the most general instance of a channel characterized by arbitrary $\mu_{\infty}, r_{\infty}, \varphi_{\infty}$, and $\Gamma$, the initial Gaussian state for which purity is best preserved in time must have a squeezing parameter $r_{0}=r_{\infty}$ and a squeezing angle $\varphi_{0}=\varphi_{\infty}+\pi / 2$, i.e., it must be antisqueezed (orthogonally squeezed) with respect to the bath. The net effect for the evolution of the purity is that the two orthogonal squeezings of the initial state and the bath cancel each other exactly, thus reproducing the optimal purity evolution of an initial nonsqueezed coherent state in a nonsqueezed thermal bath.

\section{CONCLUSIONS}

We have shown that the purity of Gaussian states for continuous variable systems can be operationally determined by the joint measurement of two conjugate quadratures. In order to perform such a measurement, the minimal, necessary, and sufficient requirement is that the measurement apparatus records data distributed according to the Husimi quasiprobability function. We have then verified by Monte Carlo simulated experiments the statistical reliability of the associ- ated measurement schemes, thus proving the possibility of an experimentally realizable characterization of the purity of Gaussian states. We have compared as well the scheme based on the $Q$ function with the one based on single-quadrature detection, and showed that the former provides a more reliable statistics. Moreover, we have derived an evolution equation for the purity of Gaussian states in noisy channels, in the case of both a thermal and a squeezed thermal bath. Our analysis shows that the purity is maximized at any given time for an initial coherent state evolving in a thermal bath, or for an initial squeezed state evolving in a squeezed thermal bath whose squeezing is orthogonal to that of the input state. We have focused our attention on the purity of singlemode Gaussian states. The time evolution of the purity for specific initial non-Gaussian states of great physical relevance can be studied, as well as the extension to Gaussian states of multimode systems, both pure and mixed. These topics are currently being explored and will be the subject of forthcoming work.

\section{ACKNOWLEDGMENTS}

The work of M.G.A.P. has been sponsored by INFM through the Project No. PRA-2002-CLON. F.I., A.S., and S.D.S. thank INFM and INFN for financial support. S.D.S. thanks the ESF COSLAB program. FI thanks the ESF BEC2000+ program.
[1] Quantum Information Theory with Continuous Variables, edited by S.L. Braunstein and A.K. Pati (Kluwer, Dordrecht, 2002), and references therein.

[2] Fundamentals of Quantum Information, edited by D. Heiss (Springer-Verlag, Berlin, 2002), and references therein.

[3] H.P. Yuen and A. Kim, Phys. Lett. A 241, 135 (1998).

[4] G.A. Barbosa, E. Corndorf, P. Kumar, H.P. Yuen, G.M. D'Ariano, M.G.A. Paris, and P. Perinotti, e-print quant-ph/0210089.

[5] F. Grosshans and P. Grangier, Phys. Rev. Lett. 88, 057902 (2002); F. Grosshans, G. Van Assche, J. Wenger, R. Brouri, N.J. Cerf, and P. Grangier, Nature (London) 421, 238 (2003).

[6] C. Silberhorn, T.C. Ralph, N. Lütkenhaus, and G. Leuchs, Phys. Rev. Lett. 89, 167901 (2002).

[7] C.M. Caves, Phys. Rev. D 23, 1693 (1981).

[8] R.S. Bondurant, P. Kumar, J.H. Shapiro, and M. Maeda, Phys. Rev. A 30, 343 (1984).

[9] B. Yurke, S.L. McCall, and J.R. Klauder, Phys. Rev. A 33, 4033 (1986).

[10] V. Chickarmane and B. Bhawal, Phys. Lett. A 190, 22 (1994).

[11] M.G.A. Paris, Phys. Lett. A 201, 132 (1995).

[12] M.S. Kim, J. Lee, and W.J. Munro, Phys. Rev. A 66, 030301(R) (2002).

[13] K. Banaszek, A. Dragan, K. Wódkiewicz, and C. Radzewicz, Phys. Rev. A 66, 043803 (2002).

[14] J. Eisert, S. Scheel, and M.B. Plenio, Phys. Rev. Lett. 89, 137903 (2002); G. Giedke and J.I. Cirac, Phys. Rev. A 66,
032316 (2002); J. Fiurasek, Phys. Rev. Lett. 89, 137904 (2002).

[15] Wang Xiang-bin, Phys. Rev. A 66, 064304 (2002).

[16] R. Filip, J. Opt. B: Quantum Semiclassical Opt. 4, 202 (2002).

[17] R. Filip and L. Mista, e-print quant-ph/0204105.

[18] A.S. Holevo, in Proceedings of the 4th International Conference on Quantum Communication, Measurement and Computing, edited by P. Kumar, G.M. D'Ariano, and O. Hirota (Plenum Press, New York, 1998).

[19] R. Simon, Phys. Rev. Lett. 84, 2726 (2000).

[20] L.-M. Duan, G. Giedke, J.I. Cirac, and P. Zoller, Phys. Rev. Lett. 84, 2722 (2000); G. Giedke, B. Kraus, M. Lewenstein, and J.I. Cirac, ibid. 87, 167904 (2001).

[21] A.K. Ekert, C.M. Alves, D.K.L. Oi, M. Horodecki, P. Horodecki, and L.C. Kwek, Phys. Rev. Lett. 88, 217901 (2002).

[22] R. Filip, Phys. Rev. A 65, 062320 (2002).

[23] M. Hendrych, M. Dusek, R. Filip, and J. Fiurasek, e-print quant-ph/0208091.

[24] G.M. D’Ariano, M.G.A. Paris, and M.F. Sacchi (unpublished).

[25] G.M. D'Ariano and M.G.A. Paris, Phys. Lett. A 233, 49 (1997).

[26] E. Arthurs and L.J. Kelly, Jr., Bell Syst. Tech. J. 44, 725 (1965).

[27] S. Stenholm, Ann. Phys. (N.Y.) 218, 233 (1992).

[28] W.L. Power, S.M. Tan, and M. Wilkens, J. Mod. Opt. 44, 2591 (1997).

[29] S.M. Barnett and P.M. Radmore, Methods in Theoretical 
Quantum Optics (Clarendon Press, Oxford, 1997).

[30] G. Adam, J. Mod. Opt. 42, 1311 (1995).

[31] D. Walls and G. Milburn, Quantum Optics (Springer Verlag, Berlin, 1994).

[32] P. Marian and T.A. Marian, Phys. Rev. A 47, 4474 (1993).

[33] V.V. Dodonov, J. Opt. B: Quantum Semiclassical Opt. 4, S98 (2002).

[34] G.M. D’Ariano, M.G.A. Paris, and M.F. Sacchi, Phys. Rev. A 62, 023815 (2000).

[35] J.H. Shapiro and S.S. Wagner, IEEE J. Quantum Electron. 20, 803 (1984); H.P. Yuen and J.H. Shapiro, IEEE Trans. Inf. Theory 26, 78 (1980).

[36] N.G. Walker and J.E. Carrol, Opt. Quantum Electron. 18, 355 (1986); N.G. Walker, J. Mod. Opt. 34, 15 (1987); Y. Lay and
H.A. Haus, Quantum Opt. 1, 99 (1989).

[37] M. Freyberger and W. Schleich, Phys. Rev. A 47, R30 (1993);

U. Leonhardt and H. Paul, Phys. Rev. 47, 2460 (1993).

[38] A. Zucchetti, W. Vogel, and D.-G. Welsch, Phys. Rev. A 54, 856 (1996); M.G.A. Paris, A. Chizhov, and O. Steuernagel, Opt. Commun. 134, 117 (1997).

[39] G.M. D’Ariano, C. Macchiavello, and M.G.A. Paris, in Quantum Communications and Measurements, edited by O. Hirota and M. Ozawa (Plenum Press, New York, 1995).

[40] J. Eisert and M.B. Plenio, Phys. Rev. Lett. 89, 097901 (2002).

[41] R. Simon, E.C.G. Sudarshan, and N. Mukunda, Phys. Rev. A 36, 3868 (1987).

[42] P. Marian and T.A. Marian, Phys. Rev. A 47, 4487 (1993). 